\title{
Estrategia tecno-didáctica para la solución de problemas de genética en estudiantes de educación a distancia
}

\author{
Fedra Lorena Ortiz Benavides ${ }^{1, a}$, Carmen Eugenia Piña López ${ }^{2, b}$ \\ ${ }^{1}$ Escuela de Ciencias Básicas, tecnología e Ingeniería. Universidad Nacional Abierta y a Distancia. Colombia \\ ${ }^{2}$ Escuela de Ciencias Básicas, tecnología e Ingeniería. Universidad Nacional Abierta y a Distancia.Colombia \\ -afedra.ortiz@unad.edu.co, ${ }^{\mathrm{b}}$ pinacarmen@gmail.com
}

[Recibido: 27 Julio 2017. Revisado: 10 Diciembre 2017. Aceptado: 12 Febrero 2018]

\begin{abstract}
Resumen: El propósito de la investigación fue comparar la efectividad de estrategias de enseñanza a partir del uso de un videojuego educativo y método de enseñanza tradicional para la resolución de problemas de genética. La muestra aleatoria consistió en 60 estudiantes matriculados al curso de Biología en una universidad colombiana en modalidad abierta y a distancia, se realizó un estudio cuantitativo con diseño cuasi experimental de comparación entre grupos aleatorizados con pos test. Los grupos tuvieron diferentes estrategias de enseñanza: Grupo uno: Modalidad semipresencial, los estudiantes desarrollaron el tema de solución de problemas de genética de manera tradicional, orientados por un tutor sin acceso a videojuego. Grupo dos: Los estudiantes utilizaron el videojuego como herramienta de aprendizaje de forma individual. Grupo tres: Los estudiantes se ejercitaron con el videojuego dentro del aula virtual acompañados de la estrategia de trabajo colaborativo. Las habilidades que se evaluaron para la resolución de problemas fueron: Comprender el problema, planificar la solución del problema, resolver el problema y formular las conclusiones. Para el análisis de los resultados se aplicaron pruebas de significancia para comprobar las diferencias entre los grupos. Los resultados demostraron que la estrategia de aprendizaje colaborativo utilizando el videojuego educativo como herramienta didáctica es más efectiva para mejorar el desempeño de los estudiantes en todas las habilidades evaluadas para solucionar problemas de genética.
\end{abstract}

Palabras clave Didáctica de la genética; problemas de genética; trabajo colaborativo; videojuegos, aprendizaje en línea.

\section{E-learning strategy for the solving of genetic problems in students of distance education}

Abstract: The purpose of the research was to compare the effectiveness of teaching strategies from the use of an educational video game and traditional teaching method for the resolution of genetic problems. The random sample consisted of 60 students enrolled in the course of Biology in a colombian university in open and distance modality, a quantitative study with quasi-experimental design of comparison between randomized groups with post-test was carried out. The groups had different teaching strategies: Group one: blended modality, the students developed the topic of solving genetic problems in a traditional way, guided by a tutor without access to videogames. Group two: Students used the video game inside the virtual classroom as a learning tool individually. Group three: Students exercised with the video game inside the virtual classroom accompanied by the collaborative work strategy. The skills that were evaluated for the resolution of problems were: Understanding the problem, planning the solution of the problem, solving the problem and formulating the conclusions. For the analysis of the results, significance tests were applied to verify the differences between the groups. The results showed that the collaborative learning strategy using the educational video game as a didactic tool is more effective to improve the performance of students in all the skills evaluated to solve genetic problems.

Keywords: Teaching of genetics problems; collaborative work; videogame; e-learning.

Para citar este artículo: Ortiz F., Piña C. (2018) Estrategia tecno-didáctica para la solución de problemas de genética en estudiantes de educación a distancia. Revista Eureka sobre Enseñanza y Divulgación de las Ciencias 15(2), 2301. doi: 10.25267/Rev_Eureka_ensen_divulg_cienc.2018.v15.i2.2301

\section{Introducción}

El diseño didáctico de un curso virtual incluye desde la innovación del ambiente virtual de aprendizaje (AVA), hasta el desarrollo de recursos didácticos de apoyo digital multimedia para 
el aprendizaje de aspectos temáticos detectados con mayor carga de dificultad por parte de los estudiantes. Una debilidad observada durante más de un periodo académico en los estudiantes del curso de biología en modalidad a distancia corresponde al aprendizaje de habilidades para la resolución de problemas de genética. De acuerdo con Ayuso y Banet (2002) la resolución de problemas constituye una de las tareas emblemáticas en la enseñanza de la genética, sin embargo, algunas investigaciones en el tema plantean como hipótesis que las dificultades en el aprendizaje del tema radican en la forma de incorporar los algoritmos utilizados en la resolución de problemas al contexto de los procesos de la genética(Thomson y Stewart 1985). Además, en la didáctica de la enseñanza para solucionar problemas de genética, se reporta una deficiencia en estrategias que consoliden las habilidades para resolver este tipo de problemas, puesto que su enseñanza requiere un alto conocimiento conceptual y de procedimientos matemáticos que resultan dificultosos para los estudiantes (Bugallo 1995).

Algunos estudios sobre estrategias de enseñanza en resolución de problemas de genética como la realizadas por Ayuso y Banet (2002) y Karagoz y Cakir (2011) reportan que la mayoría de los profesores aplican estrategias mecánicas de resolución de problemas sin tener en cuenta la conceptualización teórica de la genética. Igualmente, los estudios coincidieron en que los profesores que aplican métodos de enseñanza donde se incluyen estrategias meta cognitivas y habilidades de pensamiento superior, como la construcción de hipótesis, datos y análisis, reportan resultados más efectivos en la resolución de problemas genéticos.

La solución de problemas en ciencias es un tema que se investiga desde diversos enfoques de la psicología y que han determinado la forma de enseñar a solucionar problemas. En esta investigación se toma como referencia el enfoque cognitivo y sociocultural, por consiguiente se considera la solución de problemas como un proceso que está íntimamente ligado al desarrollo de habilidades cognitivas complejas, que tienen como objetivo encontrar la solución a una dificultad que no era inmediatamente alcanzable (Young y Freedman 2009), desde este enfoque se requiere la comprensión de la situación, la planificación de una estrategia, la capacidad para ejecutarla y analizar la respuesta en función de lo que se quiere solucionar (Mujica 2003).

Sin embargo, también se reconoce la necesidad de la interacción social para establecer puentes cognitivos que les permitan a los estudiantes pasar de estudiantes novatos a estudiantes expertos para solucionar problemas. En este sentido en la educación a distancia se tiene la necesidad de incorporar metodologías que motiven a los alumnos a ser los principales actores en el proceso de enseñanza-aprendizaje. Las tecnologías de la información y la comunicación (TICs) aparecen como protagonistas en este cambio, entre ellos el desarrollo de videojuegos. Según Hardy (2008) estos tipos de software educativos diseñados mediante simuladores basados en la resolución de problemas representan una alternativa instruccional efectiva para la enseñanza de este tipo de contenidos. Igualmente, Bernat (2006) afirma que la tendencia docente para apoyarse en los videojuegos radica en su capacidad para mejorar el rendimiento académico, debido a que la interacción con este recurso motiva y desafía al estudiante para desarrollar habilidades aplicadas a la resolución de problemas y a aprender por ensayo y error.

Para Gross (2007), esta ventaja está asociada al uso de la tecnología digital que permite interacciones centradas en el usuario, facilitan la promoción de desafíos, cooperación, compromiso, y el desarrollo de estrategias para la solución de problemas. La investigación sobre el uso educativo de videojuegos es un problema de integración entre las potencialidades del desarrollo de software y las teorías del aprendizaje.

Desde esta perspectiva, Young (2003), encontró que: a) los videojuegos deben buscar la comprensión significativa de los contenidos; b) los juegos deben estar adaptados para que los jugadores actúen en forma ampliamente cognitiva y social; c) los juegos deben entenderse 
desde la perspectiva de una teoría del aprendizaje que permita diseños instruccionales proactivos direccionados hacia dominios específicos de contenido. Además, Giménez, Pagel y Martínez (2011) aclaran que los videojuegos no se pueden encasillar como recursos únicamente para niños, sino, que pueden ser muy útiles en la educación para adultos dadas sus características lúdicas, las cuales pueden ser útiles para aprender contenidos complejos.

Por ello, la decisión pedagógica de uso de un videojuego es mucho más compleja, debido a la gran cantidad de principios didácticos involucrados para lograr que el aprendizaje sea realmente efectivo, sobre todo en aquellos conceptos que resultan abstractos como es el tema de genética (Treagust, Chittleborough y Mamialo 2002). Sin embargo, Srinivasan y Crook (2005) afirman que la instrucción multimedia en las ciencias no cumplió las expectativas en cuanto a su efectividad en el aprendizaje y que por lo tanto es necesario considerar estrategias didácticas que integradas a este tipo de recursos afecten positivamente en el desarrollo de las habilidades cognitivas.

En así que Pontes (2005) advierte que las simulaciones de fenómenos naturales resultan interesantes y ayudan a los estudiantes a resolver problemas, y que por lo tanto pueden utilizarse como instrumentos de aprendizaje significativo, siempre y cuando el profesor que los vaya a utilizar elabore previamente una guía de actividades, que permita orientar el aprendizaje y desarrollo de habilidades. En este sentido, Smaldino, Lowther y Rusell (2008) y Ortiz, Álava, Argoty y Fernández (2009), coinciden en señalar que el uso de estos recursos multimedia sin una estrategia pedagógica puede conllevar a un aprendizaje mecanicista, puesto que los estudiantes repiten los procesos sin activar sus conocimientos previos que los impulsen a un verdadero aprendizaje significativo y por el contrario estimulan el conductismo en su modelo más elemental, en el esquema de estímulo-respuesta (Jara, Candelas, Torres, Dormido, Esquembre 2009). Por esta razón hay un considerable interés en la comunidad de investigación en la enseñanza de las ciencias, para establecer el efecto de estos sistemas multimedia en la capacidad cognitiva y de solución de problemas (Mayer y Moreno 2003).

Teniendo en cuenta los argumentos anteriormente señalados, se diseñó una herramienta multimedia en formato de videojuego para solucionar problemas de genética, el cual se ejecuta dentro de una interface virtual llamada "La Granja Genogenios". (Piña y Ortiz 2014); en este contexto se presenta desafíos a los participantes en forma de problemas útiles para el mejoramiento genético de la granja. El videojuego cuenta con las siguientes secciones: a) Interfaz del videojuego, la cual presenta el menú de acceso, los personajes y los botones de actividad, b) reglas para el usuario, c) recursos didácticos de apoyo como ejemplos de aplicación de las leyes de Mendel y casos de herencia no mendeliana d) normas rápidas mendelianas y no mendelianas, e) espacio para la solución de problemas y f) solución correcta de los problemas.

El juego consta de tres niveles de acuerdo con la complejidad de los problemas planteados y pasan de un nivel inferior al siguiente solamente cuando han logrado resolver los problemas propuestos en cada nivel. En la investigación realizada por Piña y Ortiz (2014) donde se validó el videojuego "Genogenios" como recurso de aprendizaje, demostró que el 74\% de los estudiantes lo consideraron una herramienta innovadora y motivante para el aprendizaje en la resolución de problemas de genética. Sin embargo, durante este estudio no se estableció la estrategia didáctica que asociada al videojuego tenga mayor efectividad para el desarrollo de este tipo de habilidades.

Por lo tanto, el propósito de este trabajo consistió en comparar simultáneamente la efectividad de estrategias de enseñanza para la solución de problemas de genética en estudiantes de modalidad de educación a distancia: Una de interacción directa entre estudiante-profesor 
(semipresencial) y una mediada por computador utilizando como herramienta el videojuego Genogenios y éste último con dos estrategias de interacción: individual y grupal.

\section{Métodos}

Con el fin de responder la siguiente pregunta de investigación: ¿Q Qué estrategia de enseñanza es más efectiva para mejorar la resolución de problemas de genética en estudiantes de un curso de Biología bajo la modalidad de educación a distancia?, se realizó un estudio cuantitativo con un diseño cuasi experimental de comparación entre grupos aleatorizados con pos test, según lo definen Edmonds y Kennedy (2012).

\section{Contexto}

La investigación se realizó en una universidad colombiana con modalidad de educación a distancia, con proyección educativa en todas las regiones del país. El curso de Biología forma parte de la cadena curricular de diferentes programas de formación como Ingeniería de Alimentos y Regencia de Farmacia. Dentro del contenido de enseñanza del curso se encuentra la genética y resolución de problemas, siendo uno de los temas donde se encontró mayor dificultad de aprendizaje, afectando el rendimiento académico del curso.

\section{Sujetos de estudio y Muestra}

Los participantes fueron estudiantes adultos, hombres y mujeres, matriculados al curso de Biología de la universidad donde se realizó la investigación. Teniendo en cuenta que el total de estudiantes matriculados al curso de Biología fue de 80 estudiantes, se conformó para el estudio una muestra representativa de tipo pirobalística de 60 participantes con un nivel de confiabilidad del $95 \%$ y un margen de error del 5\%. La muestra es probabilística, puesto que se les envío a todos los estudiantes del curso una invitación para participar en la investigación, donde se les explico el objetivo de la misma y se les dio una fecha límite de inscripción hasta alcanzar el total de estudiantes participantes.

La conformación de los grupos de estudiantes que abordaron los problemas de genética con diferente estrategia de enseñanza se realizó a partir de un muestreo aleatorio simple, de tal manera que se distribuyeron en tres grupos hasta alcanzar un tamaño de 20 estudiantes por grupo, para un total de 60, como se indica en la Tabla 1.

Tabla 1. Conformación de grupos de estudiantes con diferentes estrategias de enseñanza. Se indica el tipo de estrategia, el número de estudiantes y la duración de la intervención.

\begin{tabular}{|l|c|c|}
\hline Tipo de estrategia de enseñanza & N. de estudiantes & Duración \\
\hline $\begin{array}{l}\text { Semipresencial - Interacción directa: Tutor-es- } \\
\text { tudiante }\end{array}$ & 20 & 15 \\
\hline $\begin{array}{l}\text { Estrategia mediada por computador con } \\
\text { interacción individual con el videojuego. }\end{array}$ & 20 & 15 \\
\hline $\begin{array}{l}\text { Estrategia mediada por computador asociada al } \\
\text { videojuego con interacción grupal en foros de } \\
\text { trabajo colaborativo en línea. }\end{array}$ & 20 & 15 \\
\hline
\end{tabular}




\section{Diseño de la investigación}

Para responder la pregunta de investigación se realizó un diseño cuasi experimental de comparación entre grupos aleatorizados. Los grupos tuvieron diferentes estrategias de enseñanza: Grupo uno: Modalidad semipresencial, los estudiantes desarrollaron el tema de solución de problemas de genética de manera tradicional, orientados por un tutor sin acceso a videojuego. Grupo dos: Los estudiantes utilizaron el videojuego como herramienta didáctica de forma individual y grupo tres: Los estudiantes se ejercitaron con el videojuego dentro del aula virtual acompañados de la estrategia de trabajo colaborativo.

El tiempo que se dio para el proceso de aprendizaje del tema de resolución de problemas de genética fue de 15 días para los tres grupos. Al finalizar las dos semanas, los estudiantes se presentaron a resolver el pos test de forma presencial. Los resultados se almacenaron en una base de datos en el software SPSS y se procedió a realizar una comparación de medias de los puntajes que obtuvieron los estudiantes dentro de los grupos en la habilidad para la resolución de problemas de genética. Luego, se realizó pruebas de normalidad de kruskal Wallis con el fin de aplicar pruebas paramétricas o no paramétrica de significancia entre los tratamientos de acuerdo con los resultados de normalidad obtenidos.

\section{Variables}

La variable independiente fue la estrategia de enseñanza así: Tratamiento uno: grupo semipresencial, quiénes estudiaron el tema de genética de manera tradicional, orientados por un tutor sin acceso a videojuego; b) Tratamiento dos: grupo de estudiantes que utilizaron el videojuego dentro del aula virtual como recurso de aprendizaje y c) Tratamiento 3: grupo de estudiantes que se ejercitaron con el videojuego dentro del aula virtual acompañados de la estrategia de trabajo colaborativo.

La variable dependiente fue la habilidad de los estudiantes para resolver problemas en ciencias de acuerdo con el planteamiento de Young y Freedman (2009). En resumen, la propuesta de evaluación consta de cuatro dimensiones: Comprender el problema, planificar la solución del problema, resolver el problema y formular conclusiones.

\section{Instrumentos}

El instrumento para recolectar la información de este estudio fue un cuestionario de preguntas abiertas (Anexo 1), el cual se validó por juicio de expertos. Como criterio de selección, se optó por un grupo de cuatro profesores universitarios cuya trayectoria se caracteriza por una larga experiencia en la enseñanza de la Biología en educación a distancia y un experto en diseño de cuestionarios para evaluación de competencias. Como modalidad de evaluación a los expertos se aplicó un formulario escrito de forma individual, donde el experto debía calificar en una escala de 1 a 5 las afirmaciones, siendo 1 la valoración inferior (Anexo 2).

Los expertos evaluaron la estructura gramatical y redacción de cada uno de los ítems, la complejidad de la pregunta de acuerdo con el nivel de educación y la validez de los ítems para evaluar el desempeño de los estudiantes en cada una de las dimensiones necesarias para resolver problemas de genética. Una vez analizadas las observaciones de los expertos, se procedió a ejecutar los arreglos en el cuestionario antes de aplicarlo a los estudiantes. El Cuestionario de evaluación constó de 12 preguntas abiertas con un espacio para explicar la respuesta y solucionar el ejercicio.

Para la valoración de las respuestas del cuestionario, se tuvo en cuenta el indicador de desempeño formulado para cada dimensión como se indica en la Tabla 2. Se asignó 0,417 
puntos a cada respuesta acertada, que resulta de dividir el puntaje máximo (5 puntos) por el número de preguntas (12), a las respuestas que no alcanzaron el indicador descrito se les asignó una puntuación de cero (0).

Tabla 2. Diseño del cuestionario de evaluación. Se indica el tipo de habilidad necesaria para solucionar problemas de genética (dimensión), acción esperada por el estudiante, indicador de desempeño y valoración por cada ítem correcto.

\begin{tabular}{|c|c|c|c|c|}
\hline $\begin{array}{l}\text { Habilidad para la solución } \\
\text { de problemas de genética }\end{array}$ & Acción & $\begin{array}{l}\text { Indicador de de- } \\
\text { sempeño }\end{array}$ & Valoración & $\begin{array}{l}\text { Puntaje máximo } \\
\text { por habilidad }\end{array}$ \\
\hline \multirow[t]{2}{*}{ Comprender el problema } & \multirow[t]{2}{*}{$\begin{array}{l}\text { Identifica la in- } \\
\text { cógnita del pro- } \\
\text { blema y los datos } \\
\text { que aportan para } \\
\text { la solución }\end{array}$} & \multirow{2}{*}{$\begin{array}{l}\text { Identifica el problema } \\
\text { dentro de una de las } \\
\text { Leyes de Mendel o } \\
\text { con algún caso de he- } \\
\text { rencia no mendeliana. }\end{array}$} & $\begin{array}{l}\text { Ítem correc- } \\
\text { to }=0.417 \text {. }\end{array}$ & \multirow[t]{2}{*}{5 puntos. } \\
\hline & & & $\begin{array}{l}\text { Numero de } \\
\text { ítems. } 12 .\end{array}$ & \\
\hline \multirow[t]{2}{*}{$\begin{array}{l}\text { Planificar la solución del pro- } \\
\text { blema }\end{array}$} & \multirow[t]{2}{*}{$\begin{array}{l}\text { Identifica los } \\
\text { conceptos impli- } \\
\text { cados en la solu- } \\
\text { ción del proble- } \\
\text { ma y los pasos a } \\
\text { seguir. }\end{array}$} & $\begin{array}{l}\text { Escoge las posibilida- } \\
\text { des de combinación } \\
\text { existente entre los } \\
\text { alelos dependiendo } \\
\text { del tipo de herencia a } \\
\text { la que corresponde el } \\
\text { problema. }\end{array}$ & $\begin{array}{l}\text { Ítem correc- } \\
\text { to }=0.417\end{array}$ & \multirow[t]{2}{*}{5 puntos } \\
\hline & & $\begin{array}{l}\text { Establece la notación } \\
\text { que va a emplear }\end{array}$ & $\begin{array}{l}\text { Numero de } \\
\text { ítems. } 12 .\end{array}$ & \\
\hline \multirow{2}{*}{$\begin{array}{l}\text { Ejecutar el plan para solucio- } \\
\text { nar el problema. }\end{array}$} & \multirow[t]{2}{*}{$\begin{array}{l}\text { Realiza el análisis } \\
\text { cualitativo ro y } \\
\text { cuantitativo de } \\
\text { las características } \\
\text { heredadas. }\end{array}$} & \multirow[t]{2}{*}{$\begin{array}{l}\text { Identifica las propor- } \\
\text { ciones matemáticas } \\
\text { con que se presenta el } \\
\text { genotipo y su corres- } \\
\text { pondiente fenotipo. }\end{array}$} & $\begin{array}{l}\text { Ítem correc- } \\
\text { to }=0.417\end{array}$ & \multirow[t]{2}{*}{5 puntos } \\
\hline & & & $\begin{array}{l}\text { Numero de } \\
\text { ítems. } 12 .\end{array}$ & \\
\hline \multirow[t]{2}{*}{ Solución del problema } & \multirow{2}{*}{$\begin{array}{l}\text { Determina la so- } \\
\text { lución del proble- } \\
\text { ma de forma co- } \\
\text { herente con el } \\
\text { planteamiento. }\end{array}$} & \multirow[t]{2}{*}{$\begin{array}{l}\text { Establece la solución } \\
\text { correcta al problema } \\
\text { planteado. }\end{array}$} & $\begin{array}{l}\text { Ítem correc- } \\
\text { to }=0.417 \text {. }\end{array}$ & \multirow{2}{*}{5 puntos } \\
\hline & & & $\begin{array}{l}\text { Numero de } \\
\text { ítems. } 12 \text {. }\end{array}$ & \\
\hline
\end{tabular}

\section{Estrategias de enseñanza}

\section{Estrategia semipresencial}

Para la interacción de enseñanza semipresencial, los estudiantes asistieron a dos encuentros tutoriales de forma tradicional con un docente durante dos fines de semana, en el primer encuentro se realizó una presentación del tema de forma magistral donde se presentaron 
ejemplos en clase y como tarea se plantearon ejercicios con diferentes niveles de complejidad para resolver en casa, de tal manera que en el segundo encuentro se espera que los estudiantes formulen sus dudas y dificultades, las cuales fueron resueltas en conjunto. Para este proceso, los estudiantes contaron con el texto guía del curso y referencias bibliográficas del tema.

\section{Estrategia mediada por computador con interacción individual con el videojuego Genogenios}

Para el grupo que interactuó de forma individual con el videojuego, se realizó una sesión tutorial donde se les entregó una guía de orientación para acceder al videojuego y como navegar en él, igualmente se les motivó para que realicen los ejercicios dentro del videojuego de acuerdo a los niveles de complejidad y utilicen los recursos didácticos que ofrece el software para reforzar su aprendizaje. Se permitió el acceso al videojuego por un tiempo de dos semanas para que cada estudiante los resolviera de manera asíncrona y lo utilizaran sin restricción para su ejercitación.

\section{Diseño del videojuego}

El videojuego "Genogenios" fue desarrollado en el año 2014 por Piña, Ortiz y Salazar. Para su diseño se realizó una triangulación entre las necesidades didácticas, los objetivos de aprendizaje y el recurso tecnológico. Cada uno de los componentes del videojuego se diseñó teniendo en cuenta la función didáctica como se indica en la Figura 1, donde se muestra el tipo de habilidad, el recurso y la acción que debe realizar el estudiante durante el juego. 


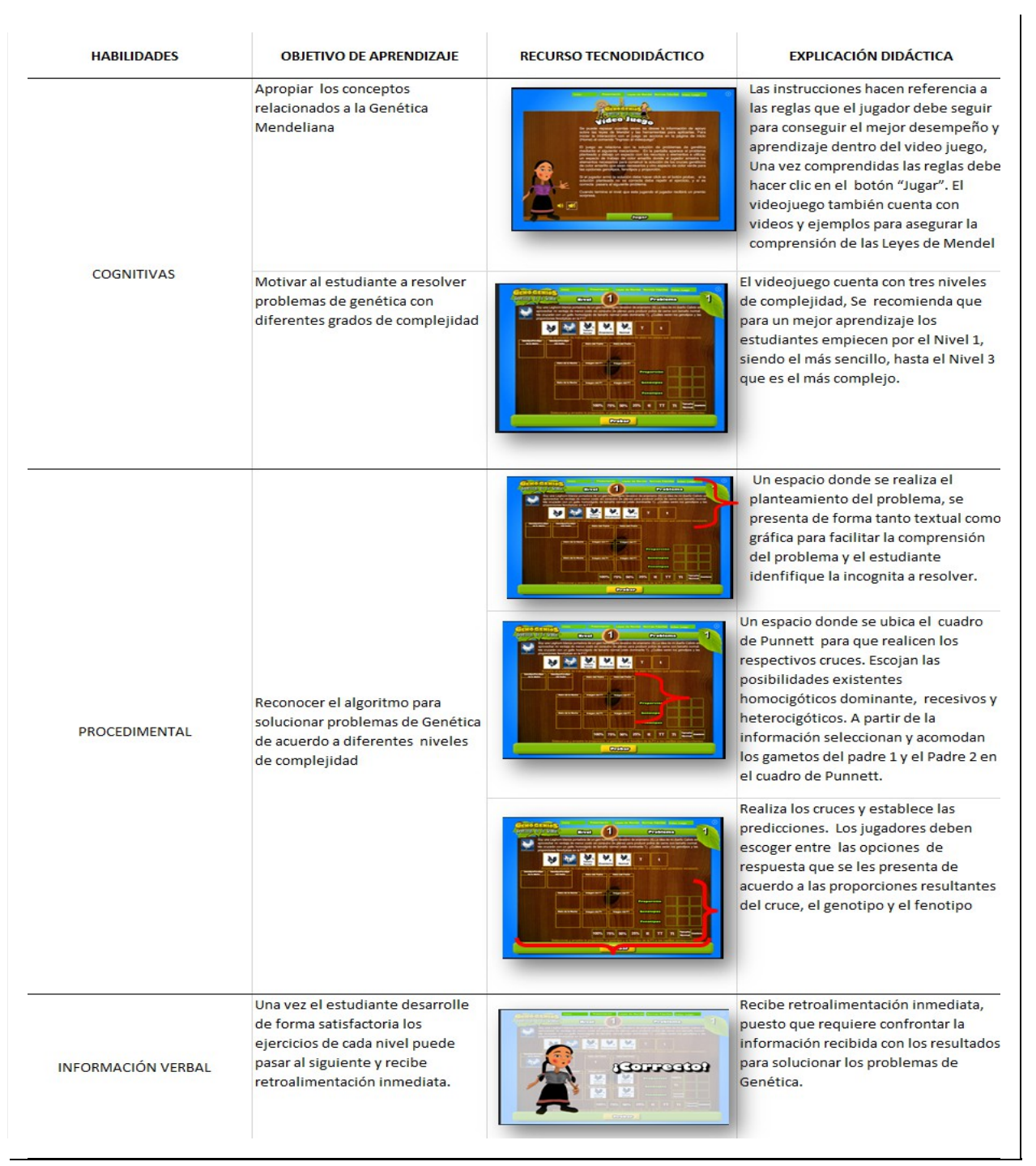

Figura 1. Diseño didáctico del Videojuego Genogenios. Fuente: Piña C., Ortiz F., (2014) Validación del videojuego Genogenios como mediador didáctico para el aprendizaje de las leyes de la herencia y su aplicación en problemas de genética. Memorias del XV Encuentro Virtual Educa- Perú. pp-301-319.

\section{Estrategia mediada por computador asociada al videojuego con interacción grupal en foros de trabajo colaborativo en línea.}

La estrategia de trabajo colaborativo consistió en formar grupos de cinco estudiantes dentro de la plataforma tecnológica donde se imparte el curso virtual de Biología. La guía de actividades se orientó en dos momentos: individual y colaborativa. Durante el momento individual los participantes además de estudiar los contenidos teóricos del tema debían realizar los ejercicios dentro del videojuego como parte de la aplicación de la teoría. Una vez desarrollados, en la fase colaborativa los estudiantes sustentaron a sus compañeros los fundamentos teóricos y procedimentales que utilizaron para solucionar el problema. Igualmente, comentaron las dificultades para resolver los problemas del videojuego. A medida que realizaron la interacción, los estudiantes debatieron la participación de sus compañeros y profundizaron en las temáticas de estudio. Al finalizar la actividad se les solicitó consolidar un informe con las participaciones individuales y las conclusiones a las que llegó el grupo. La 
interacción dentro del trabajo colaborativo estuvo abierta durante dos semanas. En la Figura 2, se indica las etapas y los momentos que se establecieron dentro de la estrategia.

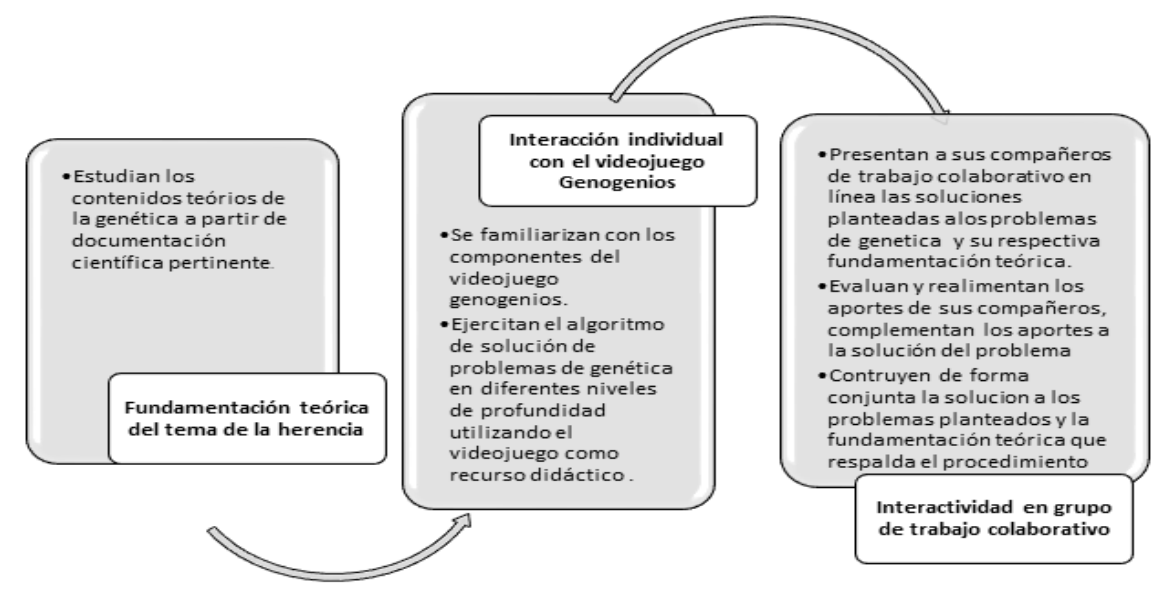

Figura 2. Esquema de la estrategia asociada al videojuego con interacción grupal en foros de trabajo colaborativo en línea para fortalecer las habilidades en la resolución de problemas de genética en estudiantes de educación a distancia.

\section{Resultados}

Con el fin de responder la pregunta: ¿Qué estrategia de enseñanza es más efectiva para mejorar la resolución de problemas de genética en estudiantes de un curso de Biología bajo la modalidad de educación a distancia? se realizó un análisis estadístico descriptivo de los resultados obtenidos en el cuestionario en cada grupo de estudiantes de acuerdo al tipo de estrategia. Los resultados de la media, la mediana y la varianza se resumen en la Tabla 3, donde se observa que los estudiantes que participaron con estrategia de interacción en foro de trabajo colaborativo presenta una tendencia hacia los niveles altos, mientras que en el grupo con interacción individual con el videojuego la tendencia se encuentra en el nivel medio y finalmente el grupo de estudiantes que participó en sesiones con modalidad semipresencial presentan una tendencia a localizarse en las puntuaciones más bajas en todas las habilidades.

Tabla 3. Estadísticos descriptivos de los resultados obtenidos en el cuestionario por los estudiantes con diferentes estrategias de enseñanza.

\begin{tabular}{|c|c|c|c|c|}
\hline \multirow{2}{*}{$\begin{array}{l}\text { Estrategia } \\
\text { de enseñan- } \\
\text { za }\end{array}$} & \multirow{2}{*}{$\begin{array}{l}\text { Tipo de habili- } \\
\text { dad (Dimensión) }\end{array}$} & \multicolumn{3}{|c|}{ Estadísticos descriptivos } \\
\hline & & Media & Mediana & Varianza \\
\hline \multirow{4}{*}{$\begin{array}{l}\text { Semipresen- } \\
\text { cial }\end{array}$} & $\begin{array}{l}\text { Comprensión del } \\
\text { problema }\end{array}$ & 1.505 & 1.400 & .607 \\
\hline & $\begin{array}{l}\text { Planificación de la } \\
\text { solución del pro- } \\
\text { blema }\end{array}$ & 1.505 & 1.400 & .607 \\
\hline & Ejecución del plan & 1.325 & 1050 & .426 \\
\hline & $\begin{array}{l}\text { Solución del pro- } \\
\text { blema }\end{array}$ & 1.125 & 1050 & .664 \\
\hline
\end{tabular}


Tabla 3. Continuación

\begin{tabular}{|c|c|c|c|c|}
\hline \multirow{2}{*}{$\begin{array}{l}\text { Estrategia } \\
\text { de enseñan- } \\
\text { za }\end{array}$} & \multirow{2}{*}{$\begin{array}{l}\text { Tipo de habili- } \\
\text { dad (Dimensión) }\end{array}$} & \multicolumn{3}{|c|}{ Estadísticos descriptivos } \\
\hline & & Media & Mediana & Varianza \\
\hline \multirow{4}{*}{$\begin{array}{l}\text { Interacción } \\
\text { individual } \\
\text { mediada con } \\
\text { el videojuego }\end{array}$} & $\begin{array}{ll}\text { Comprensión } & \text { del } \\
\text { problema } & \end{array}$ & 3.005 & 3.400 & 1.107 \\
\hline & $\begin{array}{l}\text { Planificación de la } \\
\text { solución del pro- } \\
\text { blema }\end{array}$ & 3.005 & 3.400 & 1.107 \\
\hline & Ejecución del plan & 2.855 & 2.900 & 1.107 \\
\hline & $\begin{array}{l}\text { Solución del pro- } \\
\text { blema }\end{array}$ & 2.840 & 3.150 & 1.329 \\
\hline \multirow{4}{*}{$\begin{array}{l}\text { Interacción } \\
\text { en foro de } \\
\text { trabajo cola- } \\
\text { borativo con } \\
\text { el videojuego }\end{array}$} & $\begin{array}{ll}\text { Comprensión } & \text { del } \\
\text { problema } & \end{array}$ & $4 . .050$ & 4.200 & .398 \\
\hline & $\begin{array}{l}\text { Planificación de la } \\
\text { solución del pro- } \\
\text { blema }\end{array}$ & 4.480 & 4.600 & .221 \\
\hline & Ejecución del plan & 4.460 & 4.600 & .257 \\
\hline & $\begin{array}{l}\text { Solución del pro- } \\
\text { blema }\end{array}$ & 3.950 & 4.600 & .365 \\
\hline
\end{tabular}

En el gráfico 1, se observa la diferencia de los puntajes obtenidos por los estudiantes con diferentes estrategias de enseñanza y por cada una de las dimensiones evaluadas para resolver los problemas de genética.

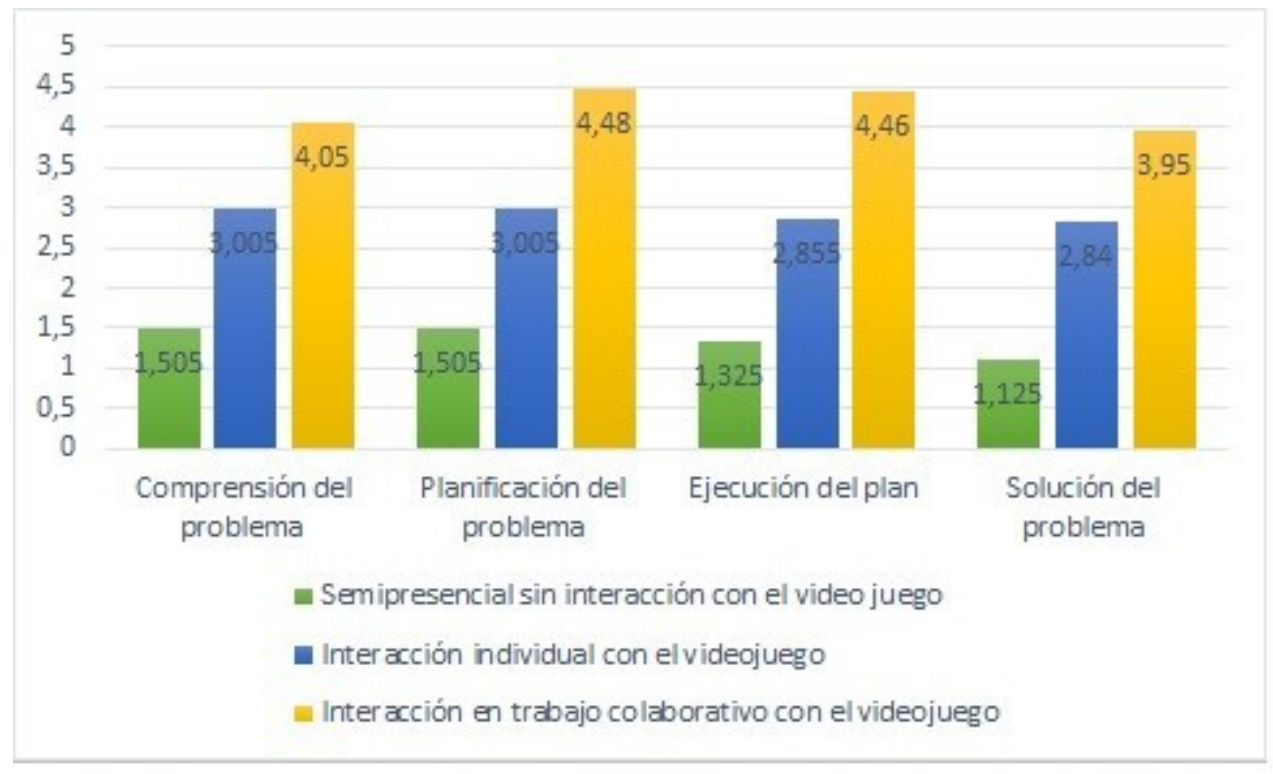

Gráfico 1. Medianas alcanzadas en las habilidades para la resolución de problemas de genética (dimensiones) por parte de los estudiantes con diferentes estrategias de enseñanza. 
Con el fin de establecer las diferencias significativas entre los grupos, se realizó pruebas de normalidad de kolmogorv Smirnov como se indica en la Tabla 4.

Tabla 4. Resultado de las pruebas estadísticas de normalidad en los diferentes grupos de estudiantes que abordaron el tema de resolución de problemas de genética a través de diferentes estrategias de enseñanza

\begin{tabular}{|l|l|l|l|}
\hline \multirow{2}{*}{ Tipo de habilidad (Dimensión) } & \multicolumn{3}{|c|}{ Kolmogorov-Smirnov $^{\mathbf{a}}$} \\
\cline { 2 - 5 } & Estadístico & gl & Sig. \\
\hline Comprensión del problema & 0,159 & 60 & 0,001 \\
\hline Planificación de la solución del problema & 0,142 & 60 & 0,004 \\
\hline Ejecución del plan para solucionar el problema & 0,138 & 60 & 0,006 \\
\hline Solución del problema & 0,166 & 60 & 0,00 \\
\hline a.Corrección de significancia de Lilliefors & & & \\
\hline
\end{tabular}

Como se puede observar en la Tabla 4, las siguientes dimensiones: Comprensión del problema, planificación de la solución del problema y solución del problema presentan $p<0.005$, por cuanto corresponde a una distribución no normal. Mientras que la dimensión ejecutar el plan para solucionar el problema proviene de una distribución de datos normal $p>0.005$.

Una vez aplicada la prueba de Kruskall Wallis para K muestras independientes, los resultados indican que existen diferencias en el nivel de la habilidad para comprender el problema entre los tres grupos estudiados $\left(X^{2}(1)=42,710, p=0,000<0.05\right)$. Los estudiantes que participaron de forma colaborativa en línea a partir del videojuego presentan el nivel para comprender los problemas de genética más alto $(\mathrm{Md}=4.2)$, seguidos de los estudiantes que realizaron la interacción con el videojuego Genogenios de forma individual $(\mathrm{Md}=3.4)$ y los que tienen el nivel más bajo para comprender los problemas formulados son los estudiantes que asistieron a tutorías de forma semipresencial sin interacción con el videojuego $(\mathrm{Md}=1.4)$.

Para la dimensión planificación de la solución del problema, el estadístico de Kruskal Wallis para $\mathrm{k}$ muestras independientes $\left(X^{2}(1)=43,452, p=0,000<0.05\right)$, indica que se presentaron diferencias significativas entre los tres grupos. Los estudiantes que participaron de forma semipresencial presentan un nivel bajo para realizar un correcto planteamiento del problema ( $\mathrm{Md}=1.4$ ), seguido de los estudiantes que realizaron la interacción individual con el videojuego Genogenios $(\mathrm{Md}=3.4)$ y los que tienen un nivel alto corresponde al grupo de estudiantes que realizaron la actividad de forma colaborativa utilizando como recurso el videojuego $(\mathrm{Md}=4.6)$.

Para la dimensión solución del problema, el estadístico de Kruskal wallis para $\mathrm{k}$ muestras independientes $\left(X^{2}(1)=38,328, p=0,000<0.05\right)$, muestra que se presentaron diferencias significativas entre los tres grupos. Los estudiantes que participaron de forma semipresencial presentan un nivel bajo en la formulación de la conclusión $(\mathrm{Md}=1.05)$, seguido de los estudiantes que realizaron la interacción individual con el videojuego Genogenios $(\mathrm{Md}=3.15)$ y los que tienen un nivel alto corresponde al grupo de estudiantes que realizaron la actividad de forma colaborativa utilizando como recurso el videojuego $(\mathrm{Md}=4.0)$. 
Puesto que los datos presentan distribución normal para la habilidad Ejecución del plan para solucionar el problema, se aplicó un análisis de varianza ANOVA. Los resultados de la prueba estadística con un nivel de significancia de 0.05, $(F=82.363, p=0.000<0.05)$, indican que existen diferencia significativa entre los tres grupos. Los estudiantes que participaron de forma semipresencial presentan un nivel bajo en la formulación de la conclusión ( $\mathrm{Md}=1.325$ ), seguido de los estudiantes que realizaron la interacción individual con el videojuego Genogenios $(\mathrm{Md}=2.855)$ y los que tienen un nivel alto corresponde al grupo de estudiantes que realizaron la actividad de forma colaborativa utilizando como recurso el videojuego $(\mathrm{Md}=4.460)$. Puesto que los resultados de la ANOVA indicaron que la estrategia de instrucción causó efecto estadístico altamente significativo en esta habilidad se aplicó la prueba post hoc de rango múltiple de Scheffe. Esta permitió la comparación de las medias entre los tratamientos, todos contra todos, de manera que cualquier diferencia existente entre un tratamiento y otro se miró reflejado en este análisis. El cuadro de comparaciones múltiples mostró que cada estrategia de instrucción se cotejó con las otras dos, con lo cual se obtuvo en cada contraste la diferencia de medias, el valor " $p$ " asociado en todos los casos resultó significativo al ser menor de 0 , en intervalo de confianza de $95 \%$.

\section{Discusión}

Los resultados, reflejan un mejoramiento creciente en todas las habilidades necesarias para resolver los problemas de genética, al pasar de la estrategia con métodos tradicionales, a la estrategia de interacción individual con el videojuego y finalmente a la estrategia de interacción colaborativa apoyada en el videojuego. El mejoramiento en la comprensión del problema, se pudo establecer porque los estudiantes relacionaron adecuadamente los conceptos asociados a la genética mendeliana tales como: cromosoma, alelo, y herencia cromosómica con los datos del problema e identificaron la incógnita que debían resolver. El anterior aspecto permitió a los estudiantes seleccionar las posibilidades de combinación de los alelos y establecer la correspondencia entre la probabilidad de los cruces con la relación numérica de los resultados en el fenotipo y genotipo; igualmente identificaron con mayor claridad aquellos casos en dónde no se cumplen las leyes de Mendel tales como la herencia ligada al sexo o codominancia, por consiguiente, los estudiantes resolvieron los problemas con mayor eficiencia.

Estos resultados se pueden explicar por cuanto el diseño del videojuego permite que los estudiantes retomen la situación problema, reflexionen sobre el mejor cruce genético, determinen con precisión los alelos participantes, ejecuten el cuadro de Punnett de forma pertinente y comprendan las operaciones matemáticas necesarias para obtener el resultado final. Desde esta perspectiva, se confirma el planteamiento realizado por Young y Freedman (2009) en el sentido que cuando un individuo es orientado para solucionar problemas debe construir varios modelos mentales para llegar a la solución, y si esto se hace durante varias sesiones de trabajo, se logra desarrollar mejores habilidades de razonamiento deductivo que cuando no se hace, como es el caso de la estrategia utilizada por el grupo uno, donde las posibilidades de ejercitación fueron más limitadas. Los resultados coinciden con los expuesto por Odadžić, Miljanović, Mandić, Pribićević, Županec (2017) quienes encontraron un efecto favorable del software educativo sobre los logros individuales de los estudiantes en el aprendizaje de la genética, en las clases de biología comparados con los métodos tradicionales.

Empero, en este estudio se observó que los recursos didácticos propios del videojuego, se potencializan durante la interacción colaborativa al establecer una comunicación consciente con los demás miembros del grupo, puesto que esta actividad exige construir postulados, comprender los que elaboran los otros miembros de su grupo y llegar a consensos para 
solucionar el problema. En este sentido Linn (2004) explica que la comunicación con los demás provoca estructuras cognitivas más complejas puesto que durante este proceso, la retroalimentación que realizan los demás miembros del grupo, los individuos la utilizan para perfeccionar la estructura cognitiva, pasando de un conocimiento abstracto y descontextualizado a un conocimiento más generalizado y listo para ser usado en una situación específica o actividad de transferencia.

Estos resultados coinciden con los logrados por Jara et al. (2009) quienes, al validar un laboratorio virtual de física en ambientes colaborativos, encontraron que la comunicación que se presenta durante el proceso de aprendizaje, supera la comunicación en prácticas tradicionales y por tanto resultan ser espacios muy deseados en la educación a distancia que permiten al usuario compartir experiencias prácticas mientras trabajan en colaboración a través de Internet. Igualmente, el estudio realizado por Potryala (2003) demostró que los métodos aplicados y las ayudas didácticas, entre otros, influyen en la correcta comprensión de la información que constituye la base del conocimiento sobre la herencia. Además, este estudio demostró que la aplicación de la enseñanza asistida por computadora asociada con métodos activos de aprendizaje, mejoran los resultados en comparación con los obtenidos al usar métodos de enseñanza tradicionales. A la vez, el estudio realizado por Ortiz y Fernández (2016), demuestra que la estrategia colaborativa en ambientes virtuales de aprendizaje orientada hacia el debate y formulación de consensos para solucionar problemas favorecen el aprendizaje conceptual y procedimental.

Otro factor a considerar en la educación a distancia es el relacionado con el nivel de complejidad de los temas para algunos estudiantes, por con siguiente, es necesario facilitar la interacción tanto con el contenido como también con los compañeros inscritos en el curso con el fin de propiciar un aprendizaje profundo (Zhenguo et al, 2010). Esto podría explicar el hecho de que los estudiantes que tuvieron acceso al videojuego y trabajaron en conjunto con sus compañeros para resolver los problemas, obtuvieron un mejor desempeño en el cuestionario que aquellos estudiantes que no lo hicieron y que recibieron instrucción manejando únicamente los recursos que utilizó el tutor de forma presencial que, aunque cumplen una función informativa no son interactivos. La interacción, según Fainholc (2010) genera aprendizaje significativo y desarrolla habilidades cognitivas más elevadas, puesto que requiere utilizar todos los sentidos para desarrollarlas. Los resultados obtenidos, afirman que el diseño didáctico es la base para garantizar que la tecnología cumpla una función facilitadora del aprendizaje no solamente de conceptos sino de habilidades cognitivas y procedimentales necesarias en la enseñanza de las ciencias.

\section{Conclusiones}

Se estableció que un contenido tan difícil y abstracto como es la teoría sobre la herencia y su aplicación en la solución de problemas de genética, se vuelve más enseñable en la educación a distancia cuando se utilizan recursos interactivos como es el caso del videojuego Genogenios, cuyo diseño les permitió a los estudiantes acceder de forma repetitiva y sin limitaciones a los ejercicios; a diferencia de la enseñanza tradicional donde el acceso a los recursos es restrictivo.

Igualmente, los resultados indicaron que los estudiantes que utilizaron el videojuego acompañados de una estrategia de interacción colaborativa, mejoraron su desempeño significativamente en todas las habilidades necesarias para solucionar problemas de genética, porque además de contar con los recursos didácticos propios del videojuego, tuvieron la oportunidad para reflexionar sobre su propio proceso de aprendizaje, discutir sus conceptos con otros miembros del grupo, aclarar dudas y replantear sus conceptos a partir de la interacción con sus pares. Por consiguiente, el presente estudio demuestra la importancia de 
continuar investigando sobre el diseño de estrategias didácticas que asociadas a recursos tecnológicos interactivos potencien las habilidades cognitivas y procedimentales de los estudiantes de ciencias naturales que adelantan su formación en la modalidad abierta y a distancia.

\section{Referencias}

Ayuso G.E., Banet E. (2002) Alternativas de la Enseñanza de la Genética en Educación Secundaria. Revista Enseñanza de las Ciencias (20)1, 133-157.

Bernat A. (2006) Los videojuegos, acceso directo a las nuevas tecnologías. Revista Comunicación y Pedagogía, 216, 6-7.

Bugallo R. (1995) La Didáctica de la genética. Revista Enseñanza de las ciencias 13(3), 379-385. DOI 10.1002/cae.2042

Edmonds W. A., Kennedy T. D. (2012) An Applied Reference Guide to Research Designs: Quantitative, Qualitative, and Mixed Methods. Thousand Oaks, CA: Sage.

Fainholc B. (2010) Lectura crítica en internet: Análisis y utilización de los recursos tecnológicos en educación. Rosario: Homo Sapiens.

Giménez C., Pagés C., Martínez J. (2011) Diseño y desarrollo de un juego educativo para ordenador sobre enfermedades tropicales y salud internacional: una herramienta docente más de apoyo al profesor universitario, Revista Eureka sobre Enseñanza y Divulgación de las Ciencias 8 (2), 221-228.

Gros B. (2007) Digital Games in Education: The Design of Games-Based Learning Environments. Journal of Research on Technology in Education 40 (1), 23-38.

Hardy M. W. (2008) A comparison of simulations and traditional laboratory exercises for student learning in secondary electronics instruction. (Tesis doctoral inédita). Nova Southeastern University, Florida.

Jara C., Candelas F., Torres F., Dormido S., Esquembre F. (2009) Synchronous collaboration of virtual and remote laboratories. Computer Aplications in Engineering Education 20 (1), 124-136.

Karagoz M., Cakir M. (2011) Problem solving in genetics: conceptual and procedural difficulties. Educational Sciences: Theory \& Practice 11(3), 1668-1674.

Linn M. C. (2004) Using ICT to teach and learn science. In R. Holliman \& E. Scanlon (Eds.), Mediating science learning through information and communication technology, (pp.9-27) Londres, Inglaterra: Routledge Falmer.

Mayer R. E., Moreno R. (2003) Nine ways to reduce cognitive load in multimedia learning. Educational Psychologist, 38(1), 43-52.

Mujica J. (2003) La enseñanza problemica en la educación científica. La Habana: Ed. Cultura y Revolución

Odadžić, V., Miljanović T., Mandić D., Pribićević T., Županec, V. (2017) Effectiveness of the Use of Educational Software in Teaching Biology. Croatian Journal of Education 19(1), 11-29.

Ortiz F., Álava C., Argoty M., Fernández P. (2009) Valoración del desarrollo conceptual y de las habilidades procedimentales y de razonamiento utilizando como mediador 
didáctico un simulador para procesos de panificación. Revista de Investigaciones $U N A D$ 8(1), 161-172.

Ortiz F., Fernández P. (2016) Diseño instruccional para argumentación en línea. Interdisciplinaria. 33(2), 231-249.

Piña C., Ortiz F., (2014) Validación del Videojuego Genogenios como mediador didáctico para el aprendizaje de las Leyes de la Herencia y su aplicación en problemas de genética. Memorias del XV Encuentro Virtual Educa- Perú. 301-319.

Pontes A. (2005) Aplicaciones de las tecnologías de la información y de la comunicación en la educación científica. Segunda parte: Aspectos metodológicos. Revista Eureka sobre Enseñanza y Divulgación de las Ciencias 2(3), 330-343.

Potyrala K. (2003) Computer-aided genetics teaching, Computer Based Learning in Science. In C.P. Constantinou, Z.C. Zacharia (Eds.), New Technologies and Their Applications in Education, (pp.929-937) Nicosia, República de Chipre.

Smaldino S. E., Lowther D. L., Russell, J. D. (2008) Instructional technology and media for learning (9th ed.). Upper Saddle River, NJ: Prentice Hall.

Srinivasan S., Crooks, S. (2005) Multimedia in a science-learning environment. Journal of Educational Multimedia and Hypermedia 14(2), 151-167.

Thomson N., Stewart J. (1985) Secondary school genetics instruction: making problem solving explicit and meaningful. Journal of Biological Education 19 (1), 53-62.

Treagust D. F., Chittleborough G., Mamialo T. L. (2002) Students' understanding of the role of scientific models in learning science. International Journal of Science Education, 24(4), 357-368.

Young, M. (2003) An ecological psychology of instructional design: Learning and thinking by perceiving-acting systems. Handbook of Research on Educational Communications and Technology, 169-177.

Young H., Freedman R. (2009) Física Universitaria. (12a ed.) México. Pearson Education

Zhenguo G., Shaobin C., Yunlong Z., Yanwen L. (2010) Construction and evaluation of flash media server based collaborative virtual. Computer Applications in Engineering Education 20 (4), 579-761. 
ANEXO 1. Cuestionario.

UNIVERSIDAD NACIONAL ABIERTA Y A DISTANCIA

CURSO DE BIOLOGÍA

CUESTIONARIO DE GENÉTICA

NOMBRE DEL ESTUDIANTE:

TIPO DE INTERACCIÓN: VIRTUAL SEMIPRESENCIAL

INTERACTIVIDAD DIDÁCTICA: Individual con el videojuego. _ Foro de trabajo colaborativo

A continuación, usted encontrará 12 PREGUNTAS que se desarrollan en torno a un enunciado sobre problema de genética, usted debe solucionar el problema, a partir del conocimiento teórico y la aplicación práctica del tema. Para desarrollar el ejercicio, desarrolle los siguientes subpuntos:

\section{Pasos a desarrollar}

A. Explique el fundamento teórico del problema

B. Identifique los alelos y la notación científica que va a utilizar. Realice el procedimiento para realizar los cruces.

C. Establezca las proporciones matemáticas del fenotipo y el genotipo correspondiente.

D. Responda la pregunta del problema.

Utilice la hoja de papel adicional para realizar los procedimientos tanto cualitativos como cuantitativos necesarios para solucionar el problema.

1. 1. Al cruzar en la F1 un conejo de ojos Marrón con una coneja de ojos Rojos todos los conejos presentaron ojos marrones. Al revisar la generación F2 se encontró que 3/4 conejos presentaron ojos marrones y $1 / 4$ conejos presentaron ojos rojos. Al razonar el cruce anterior, el genotipo de los conejos de la F2 sería.

2. De acuerdo con uno de los planteamientos de Mendel, los caracteres recesivos, al cruzar dos razas 
puras, quedan ocultos en la primera generación (F1) y reaparecen en la segunda (F2). Según este planteamiento explique cuál de las siguientes afirmaciones es correcta:

a. El $100 \%$ de la descendencia presentará el mismo fenotipo

b. El 50\% de la descendencia presentarán el mismo genotipo

c. El 50\% de la descendencia presentará el mismo fenotipo

d. El 100\% de la descendencia presentará el mismo genotipo

3. Felipe quien tiene grupo sanguíneo BO se casó con Carmen quien tiene como grupo sanguíneo O. Los dos están esperando su primer hijo (a). ¿Cuál será el tipo de sangre de su hijo?

4. Se cruzan tomates rojos híbridos y de tamaño normal homocigóticos con la variedad amarilla enana. ¿Qué proporción de los tomates rojos que salen de la F2, serán enanos?

5. En el hombre la fragilidad de los huesos está determinada por un alelo dominante. Carlos de huesos normales, se casa con Rosa de huesos frágiles, cuya madre tenía huesos normales. ¿Cuál es el genotipo de Rosa?, ¿Qué probabilidad tienen de que sus hijos tengan huesos normales?

6. La primera Ley de Mendel o de la uniformidad establece que, si se cruzan dos líneas puras para un determinado carácter, los descendientes de la primera generación son todos iguales entre sí (igual fenotipo e igual genotipo) e iguales (en fenotipo) a uno de los progenitores.

Al aplicar la primera ley de Mendel en un cruce entre un toro Hereford con cara pigmentada y una vaca Angus con cara de color blanco, se obtiene una F1 toda con color de la cara blanco. ¿Cuál es el genotipo de los padres?

7. En una misma población de cierta ave se establecieron los genotipos de los genes g y c que dan origen a los fenotipos de tamaño y forma de pico respectivamente. Estos genes se comportan de acuerdo con las leyes de dominancia, recesividad y segregación independiente de Mendel. Los resultados obtenidos se muestran en la tabla:

\begin{tabular}{|l|l|}
\hline \multicolumn{1}{|c|}{ Fenotipo } & \multicolumn{1}{|c|}{ Genotipo } \\
\hline Pico grande agudo & GGcc ó Ggcc \\
\hline Pico corto curvo & ggCC ó ggCc \\
\hline Pico grande curvo & GgCc \\
\hline
\end{tabular}

En un experimento en el que algunas aves fueron apareadas, absolutamente todos los polluelos obtenidos tenían el genotipo GgCc presentando un pico grande y curvo. ¿Cuál es el genotipo y el fenotipo de los padres?

8. Se cruzaron plantas de piña dulces con plantas de piña ácidas. La F1 obtenida fue de $100 \%$ de piñas dulces. Luego en la F2 se obtuvieron 38 plantas de piña dulce y 12 de piña ácida. Determine cuántas plantas dulces son homocigotos y cuántos heterocigotos.

9. La Fibrosis quística del páncreas, es una enfermedad hereditaria recesiva. María es portadora pero no padece la enfermedad, su esposo Juan padece la enfermedad. Ellos tienen cinco hijos, según los datos anteriores conteste: ¿cuántos de sus hijos serán sanos, y cuántos enfermos?

10. Para diferenciar dos individuos, uno homocigoto de otro heterocigótico, que presenten el mismo fenotipo, se cruzan los dos individuos con otro individuo de carácter recesivo. Al finalizar se podría llegar a la conclusión que uno de los progenitores es homocigoto si los hijos del homocigoto son:

11. Al cruzar dos moscas negras se obtiene una descendencia formada por 216 moscas negras y 72 blancas. Se sabe que el color negro es dominante y el color blanco es recesivo. Con los datos anteriores se pretende saber: ¿Cómo será la descendencia de F1?

12. La línea frontal del pelo es una característica que pueden presentarse de dos formas, una de ellas en forma continua y la otra en forma de pico conocido como pico de viuda. Gustavo quien tiene la línea frontal de forma continua se casa con Camila quien presenta pico de viuda y todos sus hijos presentan pico de viuda. Roberto quien tampoco presenta pico de viuda se casa con Martha quien si lo presenta y para su caso 4 de sus hijos tiene el pico de viuda y otros 4 no lo tienen. ¿Cuál es la diferencia entre el Genotipo de la pareja Gustavo-Camila y Roberto-Martha?

\section{GRACIAS}




\section{ANEXO 2 \\ Estrategia tecno- didáctica para la resolución de problemas de Genética.}

RÚBRICA DE EVALUACIÓN DEL POSTEST

\section{Instrucciones}

Este instrumento está dirigido a evaluar y posteriormente corregir sí hubiere lugar el pos test que se aplicará durante el proceso de ejecución del experimento didáctico y obtener los resultados correspondientes.

El cuestionario corresponde a 12 preguntas abiertas con las cuales se pretende evaluar las siguientes habilidades para resolver problemas de genética.

\begin{tabular}{|c|c|c|c|}
\hline Habilidad cognitiva & Acción & Indicador & $\begin{array}{l}\text { Número de } \\
\text { preguntas }\end{array}$ \\
\hline $\begin{array}{l}\text { Comprensión del } \\
\text { problema }\end{array}$ & $\begin{array}{l}\text { Identifica la incógnita } \\
\text { del problema y los datos } \\
\text { que se aportan }\end{array}$ & $\begin{array}{l}\text { Fundamenta el problema con } \\
\text { una de las leyes de Mendel o con } \\
\text { algún caso de herencia no } \\
\text { mendeliana. }\end{array}$ & 12 ítems \\
\hline $\begin{array}{l}\text { Planificar la solución } \\
\text { del problema }\end{array}$ & $\begin{array}{l}\text { Identifica los principios } \\
\text { y conceptos implicados } \\
\text { y establece los pasos a } \\
\text { seguir }\end{array}$ & $\begin{array}{l}\text { Escoge las posibilidades de } \\
\text { combinación existentes entre los } \\
\text { alelos dependiendo del tipo de } \\
\text { herencia Mendeliana o no } \\
\text { Mendeliana a la que corresponde } \\
\text { el problema y establece la } \\
\text { notación que va a emplear. }\end{array}$ & 12 ítems \\
\hline $\begin{array}{l}\text { Ejecutar el plan para } \\
\text { la solución del } \\
\text { problema }\end{array}$ & $\begin{array}{l}\text { Realiza el análisis } \\
\text { cualitativo y } \\
\text { cuantitativo, de tal } \\
\text { manera que determina } \\
\text { en qué medida un factor } \\
\text { aparece con mayor } \\
\text { frecuencia para } \\
\text { determinar la } \\
\text { probabilidad y las } \\
\text { proporciones } \\
\text { matemáticas }\end{array}$ & $\begin{array}{l}\text { Identifica la probabilidad con } \\
\text { que se presenta el genotipo y su } \\
\text { correspondiente fenotipo. }\end{array}$ & 12 ítems \\
\hline
\end{tabular}




\begin{tabular}{|c|c|c|c|c|c|c|}
\hline \multirow[t]{2}{*}{$\mathbf{N}^{\mathbf{o}}$} & \multirow[t]{2}{*}{ Afirmaciones para calificar el instrumento } & \multicolumn{5}{|c|}{ Calificación } \\
\hline & & 1 & 2 & 3 & 4 & 5 \\
\hline 1 & $\begin{array}{l}\text { El instrumento evaluado considera los factores relevantes para } \\
\text { indagar respecto a las habilidades para la resolución de problemas } \\
\text { de genética que se pretende evaluar: (comprensión del problema, } \\
\text { planificación de la solución, ejecución del plan y solución del } \\
\text { problema) }\end{array}$ & & & & & \\
\hline 2 & $\begin{array}{l}\text { Las preguntas presentan una contextualización clara que permite } \\
\text { la comprensión de la pregunta y de lo que se pretende evaluar }\end{array}$ & & & & & \\
\hline 3 & $\begin{array}{l}\text { La redacción del ítem contiene expresiones y palabras que son } \\
\text { conocidas por el sujeto (estudiante universitario) del cual se va a } \\
\text { obtener información con el instrumento. }\end{array}$ & & & & & \\
\hline 4 & $\begin{array}{l}\text { La redacción que tiene cada uno de los ítems es clara, completa, y } \\
\text { es de fácil comprensión por quien lo lee, de acuerdo al nivel de } \\
\text { educación. }\end{array}$ & & & & & \\
\hline 5 & $\begin{array}{l}\text { Cada ítem tiene una redacción semántica y gramatical clara y } \\
\text { comprensible. }\end{array}$ & & & & & \\
\hline 6 & $\begin{array}{l}\text { Concluyendo, considera como experto que el instrumento es } \\
\text { adecuado para indagar lo referente a la habilidad para resolver } \\
\text { problemas de genética. }\end{array}$ & & & & & \\
\hline 7 & $\begin{array}{l}\text { El o los ítems respecto a su redacción, relación gramatical y } \\
\text { semántica son (son): }\end{array}$ & & & & & \\
\hline 8 & Mis comentarios adicionales al cuestionario son: & & & & & \\
\hline 9 & Mis comentarios adicionales al ítem $\mathrm{N}^{\circ} \ldots$, son: & & & & & \\
\hline 10 & $\begin{array}{l}\text { Mis recomendaciones para mejorar la medición del constructo } \\
\text { valorado son: }\end{array}$ & & & & & \\
\hline
\end{tabular}

